\title{
Clinical outcomes in non-valvular atrial fibrillation with low CHA 2 DS 2 -VASc score after coronary stent implantation with and without hyperhomocysteinemia
}

\begin{abstract}
Objective: The aim is to demonstrate the clinical outcomes in non-valvular atrial fibrillation with low CHA 2 DS 2 -VASc score after coronary stent implantation with and without hyperhomocysteinemia.
\end{abstract}

Methods: In a retrospective, multicenter, cohort study (2011-2013) in Beijing, China ( $n=1713)$, we compared non-valvular AF patients with CHA 2 DS 2 -VASc score $=0$ or 1 receiving dual antiplatelet therapy at discharge after coronary artery stent implantation and estimated absolute risks and relative risks (RRs) of major adverse cardiac events (MACE) within 1 year. They were divided into two groups according to the plasma HCY levels before catheterization: group normal (1135 patients, $<15 \mu \mathrm{mol} / \mathrm{L}$ ) and group hHcy (553 patients, $\geq 15 \mu \mathrm{mol} / \mathrm{L})$. The primary endpoint, which was occurrence of major adverse cardiac events (MACE).

Results: After 1year follow-up, the group hHcy patients had a higher MACE rate $(p=0.017)$. The main differences between two groups were ischemic stroke $(p=0.009)$, cardiac death $(p=0.046)$ and non-fatal MI $(p=0.044)$.

Conclusions: Elevated Hcy level was independently associated with increased risk of MACE events in non-valvular atrial fibrillation patients with low CHA 2 DS 2 -VASc score after coronary artery stents implantations.

Keywords: Homocysteine - CHA 2 DS 2 -VASc score - Clinical outcomes • Atrial fibrillation

Submitted Date: 25 November 2017; Accepted Date: 04 January 2018; Published Date: 10 January 2018

\section{Background}

Homocysteine (Hcy) is a sulfhydryl containing non-protein coding amino acid formed mainly as a byproduct in the methionine metabolism and is a metabolic intermediate of the methionine-cysteine pathway, which is linked with a number of vascular diseases including hypertension, coronary artery disease, stroke, etc. The reference of tHcy in plasma is in range 5-10 $\mu \mathrm{M}$ in human. Under normal conditions, plasma Hcy concentrations do not exceed $15 \mu \mathrm{M}$ [1]. Elevation of plasma Hcy is manifested as hyperhomocysteinemia (hHcy) and has long been suggested as a risk factor for atherosclerosis and is an independent risk factor for arterial and deep venous thrombosis [2]. Positive correlation between hHcy and cardiovascular diseases led to the postulation
Xiu Feng Xie ${ }^{1,3}$, Tian Chang $\mathrm{Li}^{1,2}$

'Southern Medical University, Guangzhou-510515, China

${ }^{2}$ General Navy Hospital, Beijing-100048, China

${ }^{3}$ The Affiliated Hospital of Inner Mongolia Medical University, Hohhot-010050, China

*Author for correspondence:

E-mail: litianchang909@163.com 
that homocysteine is an independent risk factor for cardiovascular diseases [3].

Atrial fibrillation (AF) frequently occurs with coronary artery diseases and adds complexity to the selection of an appropriate antithrombotic strategy. The optimal antithrombotic treatment strategy is unresolved in patients with multiple indications. Nonvalvular $\mathrm{AF}$ is independently associated with a four- to five-fold increased risk of ischemic stroke [4]. Current evidence supports the use of both aspirin and adenosine diphosphate receptor antagonists in patients to reduce the risk of stent thrombosis or recurrent myocardial infarction (MI) [5].

In patients with non valvular AF, the CHA 2 DS 2 -VASc score is recommended for assessment of stroke risk [6]. Patients with CHA 2 DS 2 -VASc score $\geq 2$ were defined as the high risk and would be recommended to oral anticoagulation therapy. Patient with score of 0 and 1 were defined as the low risk, no antithrombotic therapy or treatment with an oral anticoagulant or aspirin may be considered, while there were a proportion of these patients to suffer from ischemic stroke. Coronary artery disease is present in $\geq 20 \%$ of the AF population. Whether uncomplicated coronary artery disease predisposes to $\mathrm{AF}$ and how $\mathrm{AF}$ interacts with coronary perfusion are uncertain [7]. Meta-analysis of several studies on folate metabolism and CVD has shown an association with hHcy and cardiovascular events, including vessel damage, atherosclerotic and thrombosis [8]. Previous studies also have reported the role of Hcy in AF patients [9].

However, the outcomes of non-valvular AF patients receiving DAPT with low CHA2DS2-VASc score after coronary stent implantation is not clear. Accordingly, we undertook this study retrospectively to describe the risk of major adverse cardiac events (MACE) in non-valvular AF patients receiving DAPT with low CHA2DS2VASc score after coronary stent implantation with and without hyperhomocysteinemia.

\section{Methods}

\section{Study cohort}

This is a retrospective study that all of the outcome data were collected post hoc. This study was conducted including all $\mathrm{AF}$ patients receiving dual antiplatelet therapy after coronary stent implantation at discharge in 7 centres in Beijing between January 2011 and December 2013 with hospital discharge diagnoses of International Classification of Diseases, Tenth Revision, Clinical Modification (ICD-10-CM) codes
410-411.Patients received coronary stent implantation this admission regardless of the treatment before. To minimize the effects of stent property differences between drug eluting stents (DES) and bare metal stents (BMS) on long-term clinical outcomes, only patients with DES implantations were included into analysis.

$\mathrm{AF}$ is defined as a cardiac arrhythmia with the following characteristics:

(1) The surface ECG shows 'absolutely' irregular RR intervals ( $\mathrm{AF}$ is therefore sometimes known as arrhythmia absoluta), i.e. RR intervals that do not follow a repetitive pattern.

(2) There are no distinct $P$ waves on the surface ECG. Some apparently regular atrial electrical activity may be seen in some ECG leads, most often in lead V1.

(3) The atrial cycle length (when visible), i.e. the interval between two atrial activations, is usually variable and, $200 \mathrm{~ms}(.300 \mathrm{bpm})$.

AF events occurring prior to admission or during the hospitalization were identified using ECGs and ICD9-CM codes 427.31 or 427.32. Patient demographics, cigarette smoking status, homocysteine and discharge medications were obtained from review of patient medical record. Body mass index (BMI) was calculated as weight (in kilograms) divided by height (in meters) squared. It was identified for history of hyperlipidemia, hypertension, heart failure (HF), chronic obstructive pulmonary disease (COPD), cancer, diabetes mellitus, stroke or transient ischemic attack prior. Glomerular filtration rate (eGFR) was estimated using the closest serum creatinine. CHA2DS2-VASc Risk Stratification Scores for Subjects With Non-valvular $\mathrm{AF}$ (the Congestive heart failure, Hypertension, Age $>75$ (doubled), Diabetes mellitus, and prior Stroke or transient ischemic attack (doubled), Vascular disease, Age 65-75, Sex category i.e. females) is a predictor of stoke and the patients may experience a higher stoke risk with higher CHA2DS2-VASc score.

\section{End point}

The primary endpoint which was occurrence of major adverse cardiac events (MACE), including ischemic stroke, cardiac death, nonfatal myocardial infarction, stroke, coronary artery revascularization. Participants were followed within 1 year following at discharge from any cause. For ischemic stroke which should be with concomitant brain imaging including magnetic resonance imaging or computed tomography, 
the ICD-9-CM codes 433.x1, 434.x1 and 436 were used to identify.

\section{Statistical analysis}

All statistical analysis was performed with SPSS19.0.Values were presented as mean \pm SD for normally distributed continuous variables, and proportions for categorical variables. The differences between continuous values were assessed using an unpaired two-tailed $t$-test for normally distributed continuous variables. Statistical significance was set at $\mathrm{p} \leq 0.05$. All Ors were given with the $95 \%$ confidence.

\section{Results}

\section{Baseline characteristics of patients}

Between 2011 and 2013, 1756 non-valvular AF incident with coronary stent implantation with CHA2DS2-VASc score $=0$ or 1 were identified, who received dual antiplatelet therapy and 25 died in hospital, leaving 1731 incident patients (mean age 62.5 \pm 9.5 years, $75 \%$ male) for our study were followed up for 1 year and 43 with missing relevant data from a follow-up, and 1688 for analysis finally.

The baseline characteristics of the study population are showed in Table 1.10 (1.1\%) had congestive heart failure defined as New York Heart Association (NYHA) class II or higher, and 120 (14\%) had dyslipidaemia. Notably, none of the patients had previous history of Stroke/TIA. 348 (20.6\%) patients had hypertension,
$190(11.2 \%)$ had diabetes mellitus, patients in HHcy group is elder and have higher proportion of current smokers than that in normal group, on the contrary, never-smokers share a lower percentage in HHcy group compared with normal group. The patients were divided into two groups according to baseline plasma total HCY level with a cut off value of $15 \mu \mathrm{mol} / \mathrm{L}$ : normal (1135 patients, $\mathrm{HCY}<15 \mu \mathrm{mol} / \mathrm{L}), \mathrm{HHcy}(553$ patients, $\geq 15 \mu \mathrm{mol} / \mathrm{L})$.

\section{One year clinical outcomes}

Within 1 year, MACE events occurred in 30 patients $30(5.41 \%), 37(3.23 \%)$ respectively. The patients in HHcy group had a higher rate of MACE than those in normal group $(5.41 \%$ vs. $3.23 \%, \mathrm{p}=0.017)$, and main differences were ischemic stroke $(2.17 \%$ vs. $0.61 \%$, $\mathrm{p}=0.009)$, cardiac death $(0.36 \%$ vs. $0.24 \%, \mathrm{p}=0.046)$ and non-fatal MI ( $1.44 \%$ vs. $0.94 \%, \mathrm{p}=0.044)$ between two group (Table 2).

\section{Hyperhomocysteinemia and CHA 2 DS 2 -VASc score}

Baseline Hcy was independently associated with ischemic strokes in non-valvular atrial fibrillation patients with low CHA 2 DS 2 -VASc score (Table 3). Elevated Hcy significantly increased the risk of MACE in patients in non-valvular atrial fibrillation patients with low CHA 2 DS 2 -VASc score (Table 4 and Figures 1 and 2).

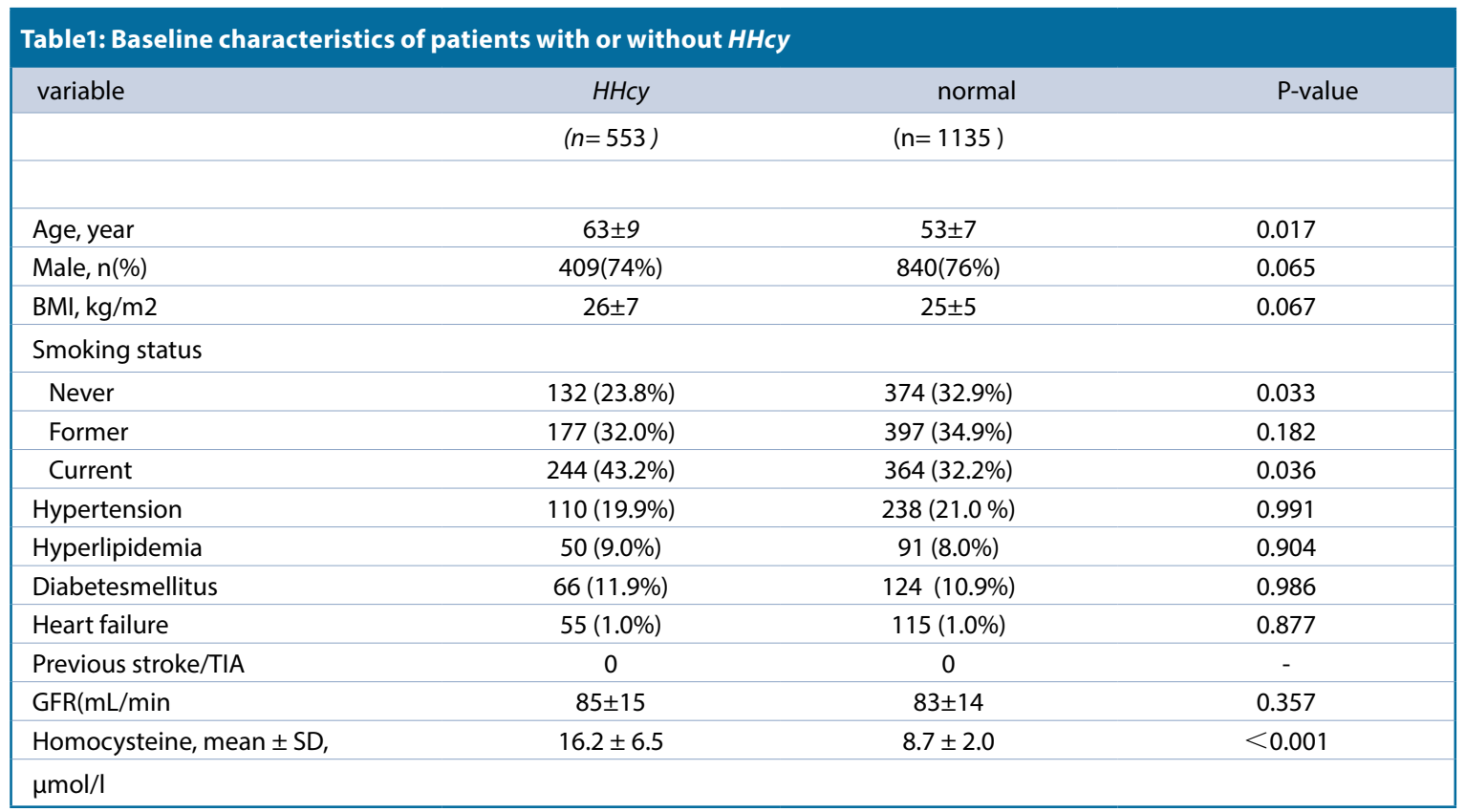

Values are expressed as mean (SD) for continuous variables and N (\%) for categorical variables. BMI: Body Mass Index; TIA: Transient Ischemic Attack 


Table 2: Clinical events during $\mathbf{1}$ year follow-up $(\boldsymbol{n}=\mathbf{1 , 6 8 8})$
\begin{tabular}{|lccc|}
\hline Number of patients & $H H c y$ & Normal & P-value \\
\hline & $(\mathrm{n}=553)$ & $(\mathrm{n}=1,153)$ & 0.009 \\
\hline Non-fatal ischemic stroke, $\mathrm{n}(\%)$ & $12(2.17)$ & $7(0.61)$ & 0.178 \\
\hline Overall mortality, $\mathrm{n}(\%)$ & $4(0.72)$ & $7(0.61)$ & 0.046 \\
\hline Cardiac, $\mathrm{n}(\%)$ & $2(0.36)$ & $3(0.24)$ & 0.129 \\
\hline Non-cardiac, $\mathrm{n}(\%)$ & $2(0.36)$ & $4(0.37)$ & 0.044 \\
\hline Non-fatal MI, $\mathrm{n}(\%)$ & $8(1.44)$ & $11(0.95)$ & 0.217 \\
\hline Coronary artery revascularization, $\mathrm{n}(\%)$ & $6(1.08))$ & $12(1.06)$ & 0.017 \\
\hline MACE, $\mathrm{n}(\%)$ & $30(5.41)$ & $37(3.23)$ & \\
\hline
\end{tabular}

\begin{tabular}{|c|c|c|c|c|c|}
\hline $\begin{array}{l}\text { CHA2DS2-VA } \\
\text { Scscore }\end{array}$ & $\mathrm{n}$ & $\begin{array}{c}\text { Ischemic stroke (HHcy vs. } \\
\text { normal) }\end{array}$ & $\begin{array}{l}\text { OR(HHcy } \\
\text { normal) }\end{array}$ & vs. $95 \% \mathrm{Cl}$ & P-value \\
\hline 0 & 912 & $1.79 \%(6 / 334)$ vs. $0 .(4 / 578)$ & 2.392 & $1.053-4.734$ & 0.043 \\
\hline 1 & 776 & $2.28 \%$ (5/219) vs. 0. (4/557) & 4.534 & $1.018-8.923$ & 0.026 \\
\hline
\end{tabular}

Table 4: Hyperhomocysteinemia and risk of MACE in different CHA2DS2 -VASc score
\begin{tabular}{|lcccccc|}
\hline CHA2DS2 -VASc score & $\mathrm{n}$ & MACE (HHcy vs. normal) & OR (HHcy normal) & vs. $95 \% \mathrm{Cl}$ & P-value \\
\hline 0 & 912 & $4.79 \%(16 / 334)$ vs. (17/578) & 2.573 & $1.008-5.236$ & 0.023 \\
\hline 1 & 776 & $7.76 \%(16 / 219)$ vs. (18/557) & 2.718 & $1.015-5.133$ & 0.016 \\
\hline
\end{tabular}

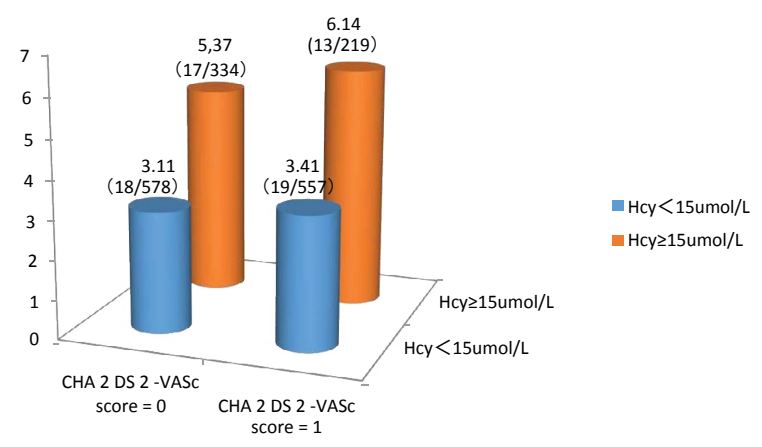

Figure 1: The prevalence of MACE according to Hcy and CHA 2 DS 2 VASc score.

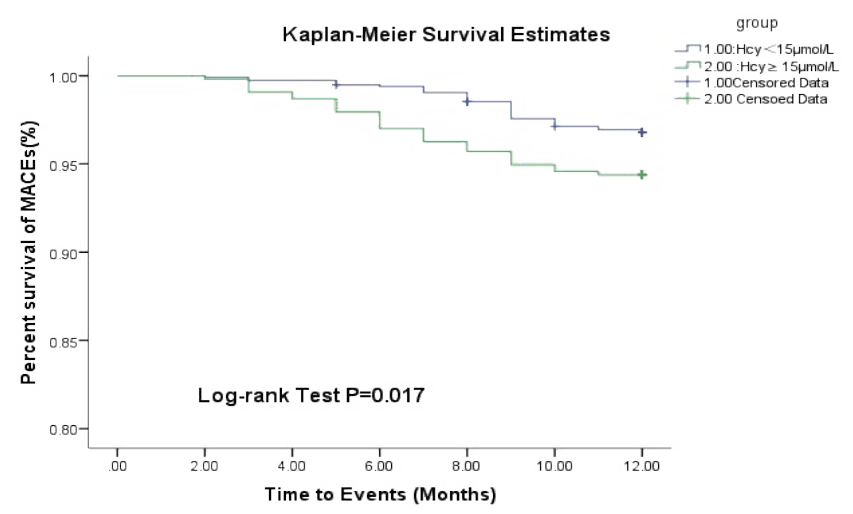

Figure 2: The MACEs-free survival by Kaplan-Meier analysis between two groups

The event-free survival rate was significantly higher in the group1 patients with lower homocysteine levels at admission $(<15 \mu \mathrm{mol} / \mathrm{L})$ (Log-rank test, $p=0.017$ ). MACE: Major Adverse Cardiac Event. 


\section{Discussion}

Now, it is widely accepted that an elevated level of Hcy (more than $15 \mu \mathrm{M}$ ) is an independent risk factor for cardio and cerebrovascular diseases. Hcy has been recognized as an independent risk factor for cardiovascular diseases (CVD) $[10,11]$. It has been estimated that there is a significant positive correlation a $2.5 \mu \mathrm{M}$ rise in plasma tHcy concentrations is associated with $10 \%$ increase in CVD risk [12].

Moreover, it was shown that increased plasma tHcy levels (above $20 \mu \mathrm{M}$ ) should be associated with ninefold increase of myocardial infarction and also with subsequent stroke risk comparing to concentration below $9 \mu \mathrm{M}$ [13]. Numerous studies have demonstrated a positive correlation between HHcy and increased inflammatory markers in acute coronary syndrome [14].

In our retrospective analysis of a multicentre registry, 1,688 AF patients with coronary artery diseases after drug eluting stents implantation with CHA 2 DS 2-VASc score of 0 and 1 were included for 1 year follow-up. The patients with hyperhomocysteinemia $(\geq 15 \mu \mathrm{mol} / \mathrm{L}$ ) had a higher MACE rate compared to normal group. After adjusting for multiple clinical, angiographic, and PCI-related variables, the association remained significant (respectively, CHA 2 DS 2-VASc score $=0: H R, 2.573$; 95\% CI: 1.008-5.236; CHA 2 DS 2-VASc score=1, HR, 2.718; 95\% CI: 1.015-5.133).

In this study, we for the first time found that elevated plasma Hcy increased the risk of MACE , demonstrating a significant predictive value for the non-valvular $\mathrm{AF}$ patients after coronary stent implantation with CHA 2 DS 2-VASc score of 0 and 1.Although there is debatable for the fact that whether Hcy is a cause or a consequence, a number of prospective cohort studies have found the moderate elevation of Hcy is an independent risk factor for cardiovascular diseases, ischaemic stroke, venous thromboembolic diseases, etc. [15].

As the studies have shown, elevated plasma HCY levels increase the risk of cardiovascular events. However, the pathophysiological mechanisms were not fully clear. As implied from basic research on the mechanism of HCY associated with vascular injury, HCY produced reactive oxygen species and caused lipid peroxidation, platelets and leukocytes activation, and increased prothrombotic factors, which resulted in vascular inflammation which may interact with $\mathrm{AF}$ and accelerate thrombus formation [16]. Furthermore, HCY causes endothelial dysfunction with impaired release of nitric oxide and vasodilatation leading to shear stress in vascular wall, which contributes to atheroma progression, and ultimately gives rise to vascular events.

Also, in further human studies, Kosokabe et al. observed a positive correlation between plasma HCY levels and atherosclerotic plaque areas in reference segments of coronary arteries, which were measured by intravascular ultrasound 6 months after the intervention [17]. This evidence supports the atherogenic propensity of HCY and also could explain our study results that worse outcomes in our patients with hyperhomocystinemia in 1 year follow-up.

In this data, the mean age in hHcy group is older than that in normal group. Similar results were found by Friedman et al. these findings suggest that elevated Hcy levels might be one of the explanations for the increased rate of stroke in elderly AF patients [18]. Hcy level increases with age, partly attributed to the increasing deficiency of vitamin B12 observed in the elderly, as result mostly by its poor absorption from food, and also due to declining renal function.

In the present study, we also found that elevated Hcy conferred a higher risk of ischemic stroke in nonvalvular AF patients with low CHA 2 DS 2 - VASc score of 0 and 1 . Similar results were found by Marcucci et al. studied $310 \mathrm{AF}$ patients on oral anticoagulant treatment and observed elevated Hcy levels were an independent risk factor for ischaemic events in non-valvular $\mathrm{AF}$ [19]. In present study, compared with CHA 2 DS 2 -VASc score of 1, it showed higher OR value and more significant difference in CHA 2 DS 2 -VASc score of 0. The difference might lie in the baseline characteristics of patients and the sample size.

Atrial fibrillation is associated with increased risk of stroke. At present, the CHA 2 DS 2 -VASc score is a widely used and well validated clinical risk prediction tool for ischemic stroke in non-valvular AF. However, it provides only modest discrimination of risk for individual patients with $\mathrm{C}$-statistic that range from 0.549 to 0.638 [20]. The clinical significance of the present study was to identify individuals at high risk of ischemic stroke who had a low stroke risk at baseline according to CHA 2 DS 2 -VASc score. It is indicated that an improved decision should be support in the care of these relatively complicated condition.

Some limitations of our study should be considered. Firstly, it is the retrospective nature of the study. Secondly, whether there is a dose dependent association between HCY levels and risks for MACE should be analyzed. 


\section{Conclusion}

In conclusion, our findings manifested that nonvalvular AF patients with hyperhomocysteinemia after coronary stent implantation had higher risk of MACE, especially ischemic stroke in spite of low CHA2DS2VASc score. The next step will be to verify the clinical outcomes over a long follow-up period.

\section{Authorship Statement}

Experiment was designed and data was analyzed by Li Tianchang and Xie Xiufeng written the article.

\section{Funding}

There is no specific funding.

\section{Conflict of Interest}

All the authors report no conflicts.

\section{Data Sharing Statement}

No additional unpublished data are available.

Executive summary

Objective: The aim is to demonstrate the clinical outcomes in non-valvular atrial fibrillation with low CHA 2 DS 2 -VASc score after coronary stent implantation with and without hyperhomocysteinemia.

Methods: In a retrospective, multicenter, cohort study (2011-2013) in Beijing, China ( $n=1713$ ), we compared non-valvular AF patients with CHA 2 DS 2 -VASc score $=0$ or 1 receiving dual antiplatelet therapy at discharge after coronary artery stent implantation and estimated absolute risks and relative risks (RRs) of major adverse cardiac events (MACE) within 1 year. They were divided into two groups according to the plasma $\mathrm{HCY}$ levels before catheterization: group normal (1135 patients, $<15 \mu \mathrm{mol} / \mathrm{L})$ and group $\mathrm{hHcy}$ (553patients, $\geq 15 \mu \mathrm{mol} / \mathrm{L}$ ). The primary endpoint, which was occurrence of major adverse cardiac events (MACE).

Results: After 1year follow-up, the group hHcy patients had a higher MACE rate $(p=0.017)$. The main differences between two groups were ischemic stroke $(p=0.009)$, cardiac death $(p=0.046)$ and non-fatal MI $(p=0.044)$.

Conclusions: Elevated Hcy level was independently associated with increased risk of MACE events in non-valvular atrial fibrillation patients with low CHA 2 DS 2 -VASc score after coronary artery stents implantations.

\section{References}

1. Seshadri S, Beiser A, Selhub J, Jacques PF, Rosenberg IH, D'Agostino RB, et al. Plasma homocysteine as a risk factor for dementia and Alzheimer's disease. $N$ Engl J Med 346: 476-483 (2002).

2. Herrmann M, Whiting MJ, Veillard AS, Ehnholm C, Sullivan DR, Keech AC, et al. Plasma homocysteine and the risk of venous thromboembolism: insights fromthe FIELD study. Clin Chem Lab Med 50: 2213-2219 (2012).

3. McCully KS. Hyperhomocysteinemia and arteriosclerosis: historical perspectives. Clin Chem Lab Med 43: 980-986 (2005).

4. Benjamin EJ, Blaha MJ, Chiuve SE, Cushman M, Das SR, Deo R. Heart disease and stroke statistics e2012 update: a report from the American Heart Association. Circulation 135: e146 (2017).

5. D’Ascenzo F, Bollati M, Clementi F, Castagno D, Lagerqvist B, de la Torre Hernandez JM, et al. Incidence and predictors of coronary stent thrombosis: evidence from an international collaborative meta-analysis including 30 studies, 221,066 patients, and 4276 thromboses. Int J Cardiol 167: 575-584 (2013).

6. Lip GY, Nieuwlaat R, Pisters R, Lane DA, Crijns HJ. Refining clinical risk stratification for predicting stroke and thromboembolism in atrial fibrillation using a novel risk factorbased approach: the euro heart survey on atrial fibrillation. Chest 137: 263-272 (2010)

7. Malinow MR, Nieto FJ, Szklo M, Chambless LE, Bond G. Carotid artery intimal-medial wall thickening and plasma homocyst(e)ine in asymptomatic adults. The atherosclerosis risk in communities study. Circulation 87: 1107-1113 (1993).

8. Refsum H, Ueland PM, Nygard O, Vollset SE. Homocysteine and cardiovascular disease. Anпu Rev Med 49: 31-62 (1998).

9. Friedman HS. Serum homocysteine and stroke in atrial fibrillation. Ann Intern Med 134: 253-254 (2001).

10. McCully KS. Vascular pathology of homocysteine: Implications for pathogenesis of arteriosclerosis. Am. J. Pathol 56: 111-128 (1969).

11. Clarke R, Daly L, Robinson K, Naughten E, Cahalane S, Fowler B, et al. Hyperhomocysteinemia: An independent risk factor for vascular disease. N Engl J Med 324: 1149-1155 (1991).

12. Williams KT, Schalinske KL. Homocysteine metabolism and its relation to health and disease. Biofactors 36: 19-24 (2010).

13. Manolescu BN, Oprea E, Farcasanu IC, Berteanu M, Cercasov C. Homocysteine and vitamin therapy in stroke prevention and treatment: A review. Acta Biochim Pol 57: 467-477 (2010).

14. El Oudi M, Bouguerra C, Aouni Z, Mazigh C, Bellaaj R, Machghoul S. Homocysteine and inflammatory biomarkers plasma levels, and severity of acute coronary syndrome. Ann Biol Clin (Paris) 69: 175-180 (2011).

15. Homocysteine Studies Collaboration. Homocysteine and risk of ischemic heart disease and stroke. JAMA 288: 2015-2222 (2002).

16. Ridker PM, Hennekens CH, Selhub J, Miletich JP, Malinow MR, Stampfer MJ. Interrelation of hyperhomocyst( e)inemia, factor v leiden, and risk of future venous thromboembolism. Circulation 95: 1777-1782 (1997).

17. Kosokabe T, Okumura K, Sone T, Kondo J, Tsuboi H, Mukawa $\mathrm{H}$, et al. Relation of a common methylenetetrahydrofolate reductase mutation and plasma homocysteine with intimal hyperplasia after coronary stenting. Circulation 103: 2048-2054 (2001). 
Clinical outcomes in non-valvular atrial fibrillation with low CHA 2 DS 2 -VASc score after coronary stent Research Paper implantation with and without hyperhomocysteinemia

18. Friedman HS. Serum homocysteine and stroke in atrial fibrillation. Ann Intern Med 134: 253-254 (2001).

19. Marcucci R, Betti I, Cecchi E, Poli D, Giusti B, Fedi S . Hyperhomocysteinemia and vitamin B6 deficiency: new risk markers for nonvalvular atrial fibrillation? Am Heart J 148:456461 (2004).

20. Hijazi Z, Oldgren J, Siegbahn A, Granger CB, Wallentin L. Biomarkers in atrial fibrillation: a clinical review. Eur Heart J 34 : 1475-1480 (2013). 